\title{
OPEN Leucyl-tRNA synthetase deficiency systemically induces excessive autophagy in zebrafish
}

Masanori Inoue ${ }^{1,2}$, Hiroaki Miyahara ${ }^{3}$, Hiroshi Shiraishi ${ }^{1}$, Nobuyuki Shimizu $^{1}$, Mika Tsumori ${ }^{2}$ Kyoko Kiyota ${ }^{2}$, Miwako Maeda ${ }^{2}$, Ryohei Umeda4 ${ }^{4}$, Tohru Ishitani ${ }^{5}$, Reiko Hanada ${ }^{4}$, Kenji Ihara ${ }^{2 \varpi}$ \& Toshikatsu Hanada ${ }^{1 \bowtie}$

Leucyl-tRNA synthetase (LARS) is an enzyme that catalyses the ligation of leucine with leucine tRNA. LARS is also essential to sensitize the intracellular leucine concentration to the mammalian target of rapamycin complex 1 (mTORC1) activation. Biallelic mutation in the LARS gene causes infantile liver failure syndrome type 1 (ILFS1), which is characterized by acute liver failure, anaemia, and neurological disorders, including microcephaly and seizures. However, the molecular mechanism underlying ILFS1 under LARS deficiency has been elusive. Here, we generated Lars deficient (lars (-) $^{- \text {) }}$ zebrafish that showed progressive liver failure and anaemia, resulting in early lethality within 12 days post fertilization. The atg5-morpholino knockdown and bafilomycin treatment partially improved the size of the liver and survival rate in lars $b^{-/-}$zebrafish. These findings indicate the involvement of autophagy in the pathogenesis of larsb $^{-/-}$zebrafish. Indeed, excessive autophagy activation was observed in larsb - $^{-/}$zebrafish. Therefore, our data clarify a mechanistic link between LARS and autophagy in vivo. Furthermore, autophagy regulation by LARS could lead to development of new therapeutics for IFLS1.

Aminoacyl-tRNA synthetases (ARSs) are essential enzymes that catalyse the ligation of amino acids to their cognate transfer RNAs (tRNAs), which is the first step in protein synthesis ${ }^{1-4}$. Leucyl-tRNA synthetase (LARS), a component of the multi-tRNA synthetase complex, is critical for charging leucine tRNA with leucine ${ }^{3}$. Furthermore, LARS has a non-canonical role as a mammalian target of rapamycin complex 1 (mTORC1)-associated protein required for amino acid-induced mTORC1 activation, indicating that LARS is not only a tRNA synthetase, but also an intracellular leucine sensor for mTORC1 signalling ${ }^{5-8}$.

The alternative functions of ARSs play a critical role in cellular homeostasis, including translation control, transcription regulation, cell migration, inflammatory responses, tumorigenesis, and cell death regulation ${ }^{9,10}$. These functions may explain the mechanisms of several human diseases caused by ARS gene mutations, including cancer, neurological disorders, and autoimmune diseases ${ }^{4,11-14}$. Biallelic mutation in the cytoplasmic LARS leads to an infantile hepatopathy called infantile liver failure syndrome type 1 (ILFS1), which is characterized by acute liver failure in the first few months and is associated with failure to thrive, anaemia, microcephaly, muscular hypotonia, and seizures ${ }^{15,16}$.

Although LARS is involved in mTORC1 pathways and its dysfunction may be responsible for ILFS1 pathology, the function of LARS in vivo has remained elusive. Previous research using a Lars loss of function $\left(\right.$ lars $\left.b^{-/-}\right)$ zebrafish model revealed that the mutant zebrafish exhibit a phenotype similar to that of ILFS1 ${ }^{17}$. Moreover, in contrast to a previous study showing that ablation of LARS desensitizes the mTORC1 pathway to amino acids in yeast and human cell lines ${ }^{5,6}$, the lars $^{-/-}$zebrafish shows augmented mTORC1 activation ${ }^{17}$. Furthermore, suppression of mTORC1 activation by rapamycin treatment or knockdown of mTORC1 by morpholino partially rescues the phenotype of lars $^{-/-}$zebrafish $^{17}$.

Therefore, to gain further insight into the LARS-mTORC1-autophagy circuit, we examined the involvement of autophagy in the pathogenesis of lars ${ }^{-/-}$zebrafish.

\footnotetext{
${ }^{1}$ Department of Cell Biology, Oita University Faculty of Medicine, Yufu, Oita 879-5593, Japan. ${ }^{2}$ Department of Pediatrics, Oita University Faculty of Medicine, Yufu, Oita 879-5593, Japan. ${ }^{3}$ Department of Neuropathology, Institute for Medical Science of Aging, Aichi Medical University, Aichi 480-1195, Japan. ${ }^{4}$ Department of Neurophysiology, Oita University Faculty of Medicine, Yufu, Oita 879-5593, Japan. ${ }^{5}$ Division of Cellular and Molecular Biology, Department of Homeostatic Regulation, Research Institute for Microbial Diseases, Osaka University, Osaka 565-0871, Japan. ${ }^{\circledR}$ email: k-ihara@oita-u.ac.jp; thanada@oita-u.ac.jp
} 
A

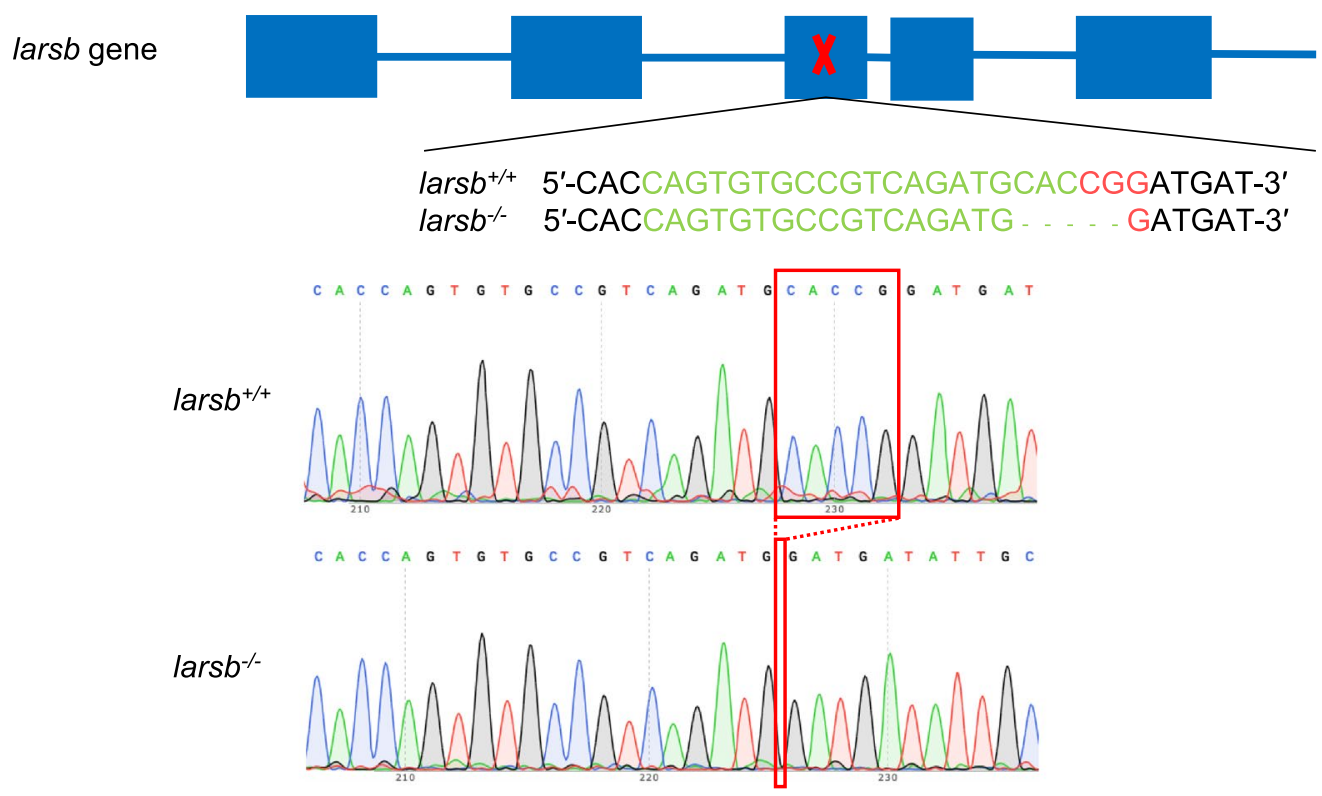

B
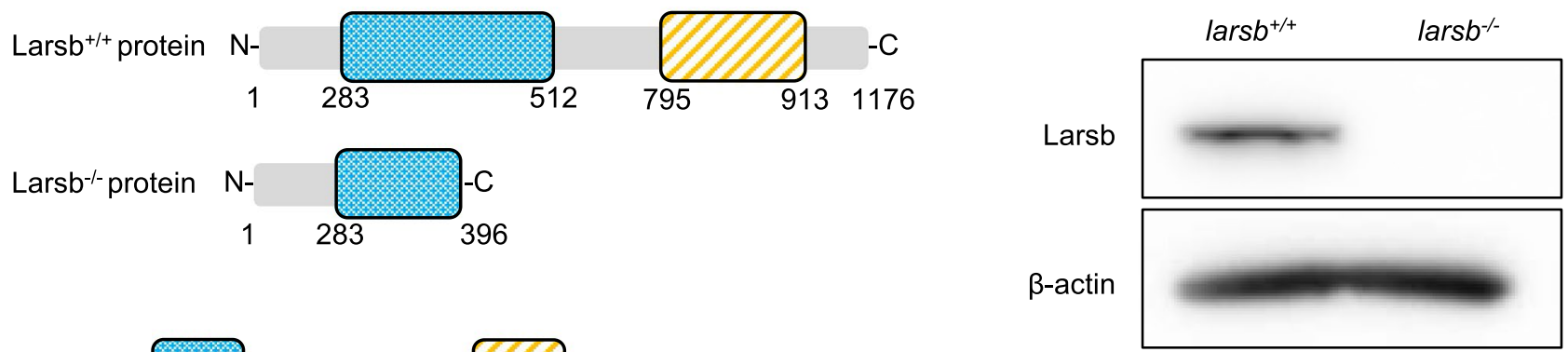

Editing domain

Anticodon binding domain

Figure 1. Construction of lars b-knockout mutant zebrafish line. (A) Diagram showing the larsb genomic locus, CRISPR/Cas9 target site, and larsb-knockout $\left(\right.$ lars $\left.^{-/-}\right)$zebrafish mutant genotype. The sgRNA target sequence is displayed in green and the PAM region in red. In the genomic sequencing analysis chromatograms, the deletion region in the mutant lars $^{-/-}$zebrafish is shown by the red box. (B) The Lars protein of lars ${ }^{-/-}$zebrafish had a missing editing domain. Western blot analysis of the Larsb protein expression in lars $b^{+/+}$and lars $b^{-/-}$zebrafish. $\beta$-actin levels served as the loading control. Larsb: leucyl-tRNA synthetase b.

\section{Results}

Generation of lars $^{-l-}$ zebrafish. To assess the function of LARS in vivo, we generated larsb-knockout $\left(\right.$ lars $\left.^{-/-}\right)$zebrafish using CRISPR/Cas9 technology ${ }^{18,19}$. Two genes, larsa and larsb encode cytosolic Lars in zebrafish, and among them, larsb shares higher homology with human LARS.

To obtain larsb mutant zebrafish, we designed the CRISPR/Cas9 target site in exon 3 of larsb (Fig. 1A), which corresponds to the editing domain of the Lars protein (Fig. 1B). Notably, most LARS gene mutations in humans occur in the editing domain ${ }^{15,16,20}$, indicating that this domain has an essential function in vivo. After screening several founders that transmitted targeted indels to the F1 progeny, we established a stable line with a frameshift mutation caused by a 5-bp deletion (Fig. 1A). Western blotting confirmed a complete lack of the Lars protein in lars $b^{-/-}$larvae (Fig. 1B). Furthermore, we performed a quantitative PCR assay to analyze the mRNA levels encoding the proteins responsible for the canonical function of Leucyl-tRNA synthetase, Larsa, Larsb, and Lars2 (Supplementary Fig S1). There was almost no expression of larsa mRNA when compared with that of larsb, indicating that larsa may be a pseudogene. Meanwhile, the mRNA expression of Lars2, a mitochondrial leucyl-tRNA synthetase that can charge mitochondrial tRNA with its cognate amino acids, significantly increased in lars $^{-/-}$zebrafish. It may be that lars2 expression is induced by the larsb gene knockout via a molecular mechanism, such as nonsense-induced transcriptional compensation (NITC) ${ }^{21,22}$, resulting in the relatively mild phenotype in lars $^{-/-}$zebrafish. 
Liver defects and early lethality in lars $\boldsymbol{b}^{-/-}$zebrafish. Lars $b^{-/-}$larvae had hatching rates and timings comparable with that of $l a r s b^{+/+}$larvae. However, all larsb $b^{-/-}$larvae exhibited thinness, cardiac edema, and swim bladder deflation (Fig. 2A). All lars l $^{-/-}$larvae died between 8 and 11 days post fertilization (dpf) (Fig. 2B). Because anaemia is one of the typical symptoms in ILFS1 patients, we performed o-dianisidine staining to detect haemoglobin-containing cells in lars $b^{-/-}$larvae. As expected, the lars $b^{-/-}$larvae showed anaemia (Fig. 2C) ${ }^{15}$.

We further analysed the morphological changes in liver development by crossing $\operatorname{lars}^{-/-}$zebrafish with $\operatorname{Tg}\left[\right.$ fabp 10:mcherry] transgenic zebrafish, which express mCherry fluorescent protein specifically in the liver ${ }^{23,24}$. The livers of $l a r s b^{-/-}$larvae were significantly smaller than that of lars $b^{+/+}$larvae at $3 \mathrm{dpf}$, and showed no further development until their death $\left(\mathrm{P}<0.001\right.$; Fig. 2D,E). These data indicate the similarity of larsb ${ }^{-1-}$ zebrafish phenotype to ILFS1 due to human LARS mutation. Thus, the in vivo function of LARS seems to be conserved across zebrafish and humans.

As previously described, the primary symptoms of mutations in GARS, SARS, HARS, and other tRNA-synthetase genes are neurological defects ${ }^{4,25}$. The lars $^{-/-}$zebrafish also showed microcephaly and loss of locomotor activity (Supplementary Figs. S2A-F). These data indicate that larsb could have an essential role in the development of the neuronal system, as previously described in other tRNA synthetases ${ }^{4,25}$.

Lars deficiency induces autophagy in lars $\boldsymbol{b}^{-1-}$ zebrafish. To assess liver abnormalities in lars $b^{-/-}$ zebrafish, we performed histopathological examination. The livers of lars $b^{-1-}$ larvae drastically reduced in size compared with that of lars $^{+/+}$larvae (Fig. 3A). In addition, large vacuolations, which seemed to disappear in the cytoplasm, were observed in the livers of $l a r s b^{-/}$larvae. Some large vacuolations included a bare nucleus. These findings indicate autophagic cell death ${ }^{26}$. Indeed, microtubule-associated protein 1A/1B-light chain 3 (LC3B)-II, a standard marker of autophagosome formation, was upregulated in lars $b^{-/-}$larvae (Fig. 3B), as shown by western blotting. The selective autophagy substrate p62 was also more degraded in lars $b^{-/-}$larvae than in $l a r s b^{+/+}$larvae (Fig. 3B). However, histologically, cytoplasmic condensation, cytoplasmic blebbing, and fragmented nuclei, which indicate apoptotic cell death, were not observed in the livers of lars $b^{-/-}$larvae. These results indicate that apoptotic cell death is not induced by Lars deficiency.

Next, to examine whether autophagy is involved in liver abnormalities, we evaluated the status of autophagy by fluorescent immunostaining for Lc3b in lars $b^{-/-}$larvae under Tg[fabp10:mcherry] background. Lc3b, a downstream constituent of the autophagy pathway and participant in autophagosome formation, is widely used to monitor autophagy $y^{27}$. Although $l a r s b^{+/+}$larvae had no apparent autophagic structures in the liver, lars $b^{-/-}$larvae displayed large vacuoles, including floating nuclei and various sized dots with Lc3b immunoreactivity, thereby indicating autophagic cell death (Fig. 3C). Hepatocellular nucleophagy, showing fragmented nuclei labelled with $\mathrm{Lc} 3 \mathrm{~b}$, was also observed in the livers of $l a r s b^{-/-}$larvae. Moreover, many autophagosomal structures visualized with Lc3b were also observed in the skeletal muscles and spinal cords of lars $b^{-/-}$larvae in comparison to lars $b^{+/+}$ larvae. Thus, these data indicate that Lars deficiency induces autophagy not only in the liver, but also in the central nervous system and skeletal muscle during the early embryonic stage.

Immunoelectron microscopy analysis of lars $^{-/-}$larvae under Tg[fabp10:mcherry] background. We next assessed the ultrastructure of the liver, skeletal muscle, and spinal cord by immunoelectron microscopy. There were no overt autophagic structures in the livers, skeletal muscles, and spinal cords of lars $^{+/+}$larvae (Figs. 4A-C). However, large vacuoles in the livers of larsb $b^{-/-}$larvae were composed of numerous irregular membranous structures with immunoreactivity against both Lc3b and mCherry (Fig. 4D,G). The mCherry protein was detected using a red fluorescent protein (RFP) antibody to confirm that the Lc3b-positive cells were hepatocytes. Many irregular structures labelled with anti-Lc3b antibody, which were presumed to be autophagosomes or autolysosomes, were also observed in the muscles and spinal cords of lars $b^{-/-}$larvae compared with those of lars $^{+/+}$larvae (Fig. 4E,F,H,I). Therefore, although autophagy caused by Larsb deficiency occurred in some tissues, including the skeletal muscle and spinal cord, the liver was the most damaged tissue in larsb $^{-/-}$zebrafish.

Inhibition of autophagy partially rescues the liver defects. To verify whether the liver defects and severe developmental abnormalities in $l a r s b^{-/-}$larvae were due to autophagy, we performed a knockdown experiment using an antisense morpholino for atg5 (atg5-MO), which is essential for autophagy induction ${ }^{28}$. As a highly efficient $\operatorname{atg} 5$ knockdown in zebrafish causes abnormal neuronal development ${ }^{29}$, the amount of MO injected was estimated to achieve a knockdown efficiency of $60 \%$ (Supplementary Figs. S3A and B).

As expected, atg5-MO prevented abnormal embryonic development, such as cardiac edema and swim-bladder deflation in lars $b^{-/-}$larvae (Fig. 5A). The effect of atg5-MO on autophagy was confirmed by western blot analysis for Lc3b-II (Fig. 5B). The atg5-MO reduced the conversion of Lc3b-I to Lc3b-II, indicating an effective inhibition of autophagy.

Atg5-MO also partially rescued the liver defects in larsb $^{-/-}$larvae ((Fig. 5C,D). However, atg5-MO did not improve the survival rate of $l a r s b^{-/-}$zebrafish, presumably because of its transient effectiveness for up to 5 days after injection (Supplementary Fig. S4).

To validate whether autophagy is involved in the $l a r s b^{-/-}$phenotype, we treated $l a r s b^{-/-}$larvae with the specific autophagy inhibitor bafilomycin A1. The effect of autophagy inhibition was estimated by western blot analysis for Lc3b-II. Bafilomycin A1 augments Lc3b-II accumulation because it inhibits autophagosomal fusion and degradation ${ }^{30}$. As expected, bafilomycin A1 treatment increased Lc3b-II accumulation, indicating that it effectively inhibits autophagy in zebrafish (Fig. 5E). Bafilomycin A1 treatment also partially improved the size of the liver in larsb ${ }^{-/}$larvae (Fig. 5F,G). Notably, we observed a substantial improvement in cardiac edema after 
A

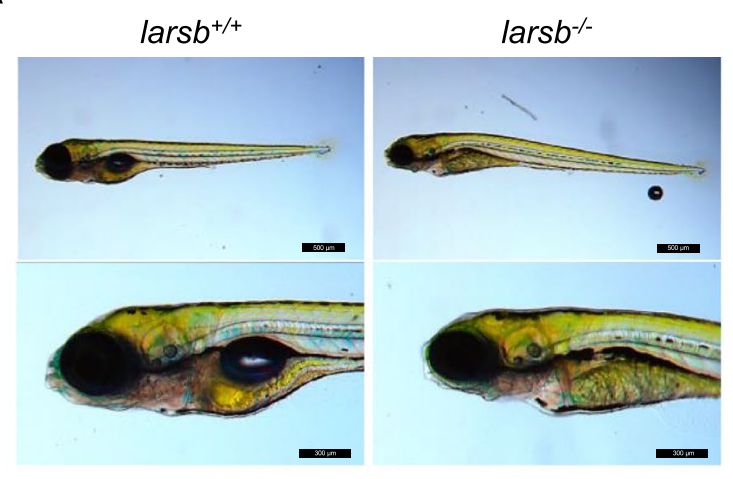

B

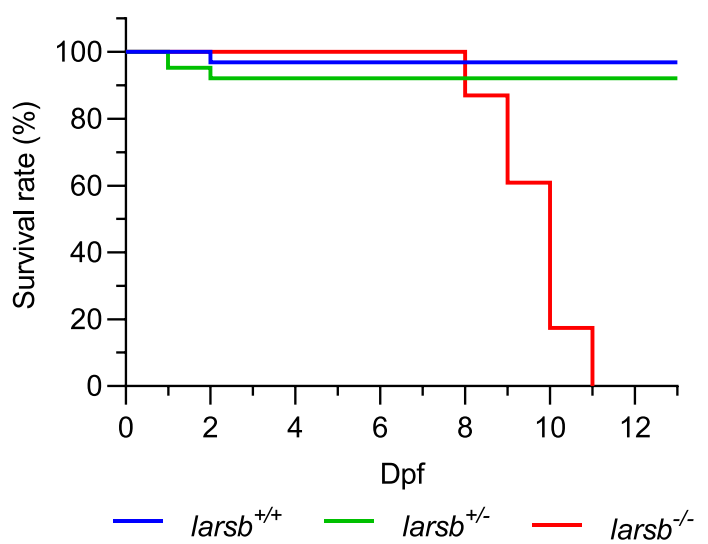

C
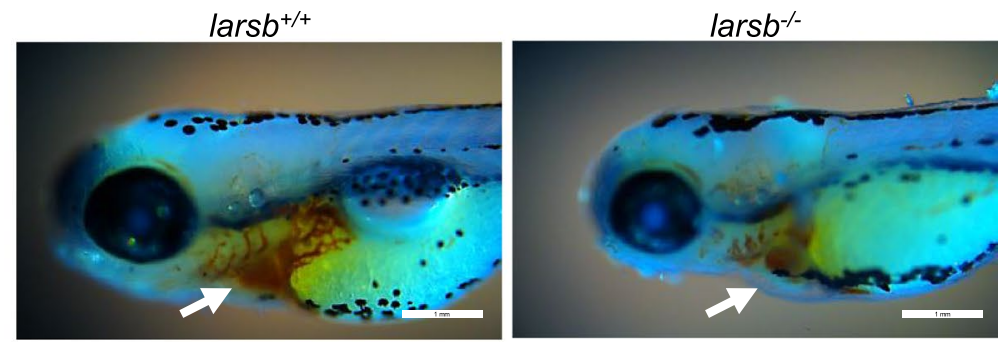

D

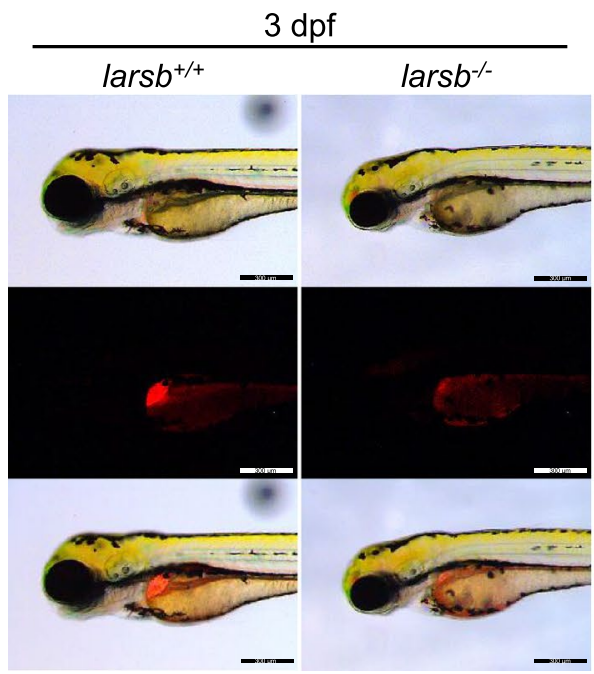

$\mathrm{E}$

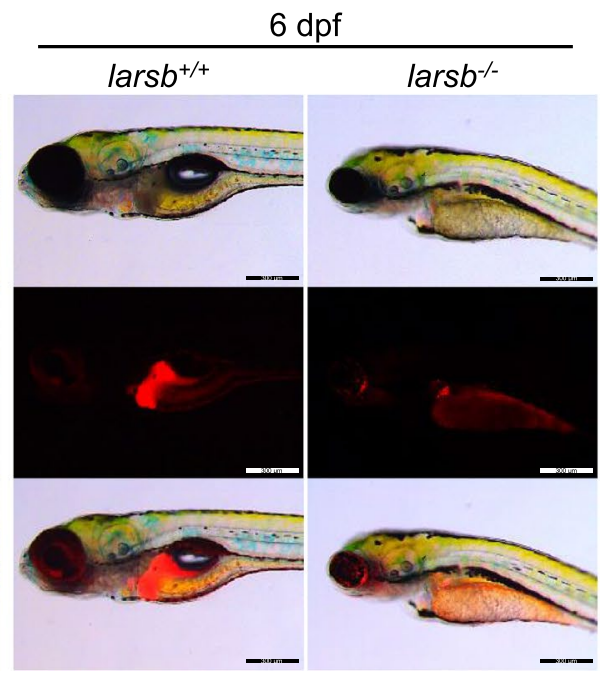

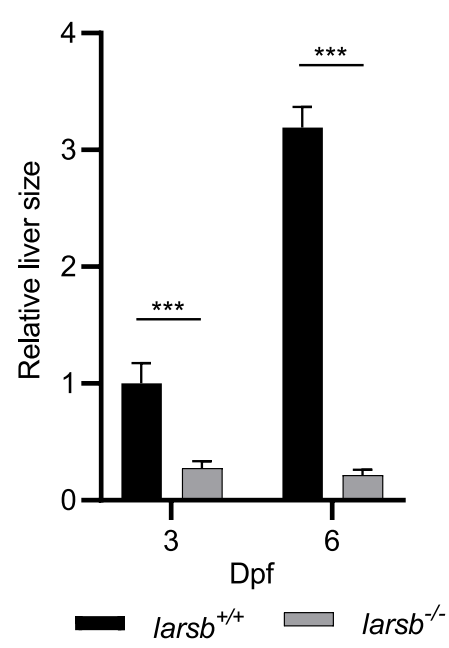

Figure 2. Larsb-knockout larvae display severe developmental phenotype and liver abnormality with early lethality. (A) Bright field lateral views of $l a r s b^{+/+}$and $l a r s b^{-/}$embryos at $96 \mathrm{~h}$ post fertilization (hpf). Scale bar: $500 \mu \mathrm{m}$ (top row) and $300 \mu \mathrm{m}$ (bottom row). (B) Kaplan-Meier survival curve of lars $b^{+/+}(\mathrm{n}=32)$, larsb $b^{+/-}$ $(\mathrm{n}=63)$, and larsb $^{-/-}(\mathrm{n}=23)$ larvae. (C) Lateral views of lars $b^{+/+}$and lars ${ }^{-/-}$embryos containing haemoglobincontaining cells (white arrows) stained with o-dianisidine at $72 \mathrm{hpf}$. (D) Morphological abnormality at 3 $\mathrm{dpf}$ and $6 \mathrm{dpf}$ in the livers of lars ${ }^{-/-}$larvae under Tg[fabp10:mcherry] background. Scale bar: $300 \mu \mathrm{m}$. (E) Quantification of liver size in larsb ${ }^{-/-}$larvae under Tg[fabp10:mcherry] background (3 dpf and $6 \mathrm{dpf}$ ). Liver sizes were evaluated using ImageJ software version $1.52 \mathrm{a}$ (https://imagej.nih.gov/ij/). $\mathrm{n}=5$ fish/group. Error bars indicate SEM. Student's t-test; ${ }^{* *} \mathrm{P}<0.001$. Statistics were calculated and the figure was produced in GraphPad software version 8 (https://www.graphpad.com/scientific-software/prism/). Larsb: leucyl-tRNA synthetase b, dpf: days post fertilization. 
treating lars $b^{-/-}$larvae with bafilomycin A1 as well as when atg5 was knocked down (Fig. 5D). The survival rate was also significantly enhanced by bafilomycin A1 treatment (Fig. $5 \mathrm{H}$ ).

To further validate the involvement of autophagy in the lars $b^{-/-}$phenotype, lars $b^{-/-}$larvae were treated with the other autophagy inhibitors, chloroquine and 3-methyladenin. Both autophagy inhibitors effectively improved the liver phenotype of lars $\mathrm{b}^{-/-}$larvae (Supplementary Figs. S5A and B). The survival rate was also significantly enhanced by chloroquine treatment (Supplementary Fig. S5C), but not 3-methyladenine, which may be linked to the high genotoxicity of 3-methyladenine ${ }^{31}$ (Fig. 5F).

In contrast, treatment with the mTORC1 inhibitor rapamycin had no effect on the survival of lars $b^{-/-}$larvae (Supplementary Fig. S6B) but retarded growth as reported previously ${ }^{32}$. This indicates that the larger liver observed in the rapamycin-treated lars $b^{-/-}$zebrafish may have been due to delayed liver phenotype progression, rather than rapamycin treatment (Supplementary Fig. S6A).

These experiments provide direct evidence that hyperactivated autophagy induced by Lars deficiency is responsible for the liver defects and an early lethality.

\section{Discussion}

In this study, we provide evidence on the in vivo function of LARS in autophagy regulation. Lars $b^{-/-}$zebrafish displayed liver failure and anaemia, a phenotype similar to ILFS1 caused by human LARS gene mutations. Histopathological analysis of lars $^{-/-}$zebrafish showed enhanced autophagy not only in the liver, but also in other tissues, including the nervous system and muscles, during early embryonic development. In addition, huge vacuolations with bare nuclei were observed in the livers of lars $^{-1-}$ zebrafish, indicating severe autophagic cell death. Inactivation of autophagy by atg 5 knockdown or bafilomycin treatment partially rescued early lethality with liver failure. These results imply that the loss-of-function mutations of LARS in ILFS1 cause severe autophagic cell death in the liver.

Previously, in vitro studies have shown that LARS induces mTORC1 activation by sensing abundant intracellular leucine concentration, thereby inhibiting autophagy ${ }^{5,6}$. In contrast, LARS dysfunction activates autophagy by inhibiting mTORC1 activity ${ }^{5,6}$. These findings indicate the essential function of LARS in regulating autophagy. Wang et al. through in vivo studies have shown that lars $^{-1-}$ zebrafish have severe liver failure and increased mTORC1 activation ${ }^{17}$. Rapamycin, an mTORC1 inhibitor, partially rescues liver failure in lars $b^{-/-}$zebrafish, $^{-}$ suggesting that hyperactivation of mTORC1 may be related to the onset of ILFS ${ }^{17}$. Therefore, there seems to be a discrepancy between the in vitro and in vivo experiments.

Our histopathological data clearly showed that $\mathrm{lars}^{-/-}$zebrafish had increased autophagy in several tissues, including the skeletal muscle and central nervous system as well as the liver. Systemic autophagy induced by Lars deficiency could explain the general symptoms of ILFS1, such as muscle hypotonia, mental retardation, and convulsions ${ }^{15,16}$. Notably, Lars deficiency-induced autophagy caused significant damage to the liver. In the muscle tissue, the mTORC1-dependent autophagy pathway is mainly regulated by insulin signalling, whereas in the liver, it is strongly regulated by amino acid concentrations ${ }^{33}$. As LARS is a leucine concentration sensor for amino acid signalling to mTORC1, LARS may play an essential role in autophagy regulation, especially in the liver.

Our experiments suggest a mechanistic link between ILFS1 and LARS loss-of-function mutations. Although rapamycin did not affect the phenotype of $l a r s b^{-/-}$larvae, the atg5-morpholino, chloroquine, and the lysosometargeting autophagy inhibitor bafilomycin A1, partially improved the survival rate and prevented liver damage. Atg5 is an important autophagy gene that forms an Atg12-Atg5-Atg16 multimetric complex and plays an essential role in autophagosome membrane expansion and completion ${ }^{34-36}$. Morpholino knockdown of atg 5 has been reported to show successful inhibition of autophagy ${ }^{37}$. Our experiment also showed the efficient knockdown of Atg5 protein expression and the suppression of Lc3b-II conversion, indicating an efficient inhibition of autophagy in vivo.

Notably, the concentration of bafilomycin, an inhibitor of vacuolar $\mathrm{H}^{+}$ATPase (V-ATPase), used in the rescue experiment was relatively low $(2.5 \mathrm{nM})$. It has a variety of effects, not only in the inhibition of autophagy, but also the inhibition of cell growth and induction of apoptosis and differentiation ${ }^{38}$. To achieve the efficient inhibitory effects on autophagic degradation, bafilomycin A1 is usually required at high concentrations (>100 nM). However, it also induces severe acidosis and secondary adverse effects in zebrafish larvae ${ }^{39}$. In fact, larvae died soon after treatment with bafilomycin A1 at $250 \mathrm{nM}$ in our experiments. Although $25 \mathrm{nM}$ of bafilomycin A1 improved the survival rate of $l a r s b^{-/-}$larvae, it did not rescue these liver defects. Therefore, we decided to conduct the rescue experiment with bafilomycin $\mathrm{A} 1$ at a concentration of $2.5 \mathrm{nM}$ to prevent toxicity for growth on larvae. Importantly, $2.5 \mathrm{nM}$ of bafilomycin A1 sufficiently accumulated Lc3b-II protein in larvae, suggesting that it effectively inhibits autophagy.

Our results suggested that suppression of excessive autophagy may rescue the symptoms of ILFS1. Of note, $\operatorname{lars}^{-/-}$zebrafish exhibited a more severe phenotype than ILFS1, although the phenotype closely resembled the symptoms of ILFS1. The exact molecular mechanism by which LARS mutation influences human ILFS1 needs to be determined using knock-in animal models, wherein a corresponding mutation is introduced into the zebrafish lars $b$ locus. Moreover, there is increasing evidence for autophagy being associated with many diseases, including sepsis, Parkinson's disease, and Alzheimer's disease ${ }^{40-42}$. Hence, autophagy regulation by LARS may lead to new therapeutics for these related disorders.

\section{Methods}

Zebrafish maintenance. Zebrafish AB genetic background larsb mutant and Tg[fabp 10:mcherry $]^{23,24}$ were raised and maintained following standard procedures. They were kept at $28-29^{\circ} \mathrm{C}$ under a 14 -h: 10 -h light:dark cycle. Embryos were collected and housed at $28.5^{\circ} \mathrm{C}$. All animal experimental procedures were performed in accordance with the institutional and national guidelines and regulations. The study was carried out in compli- 
A

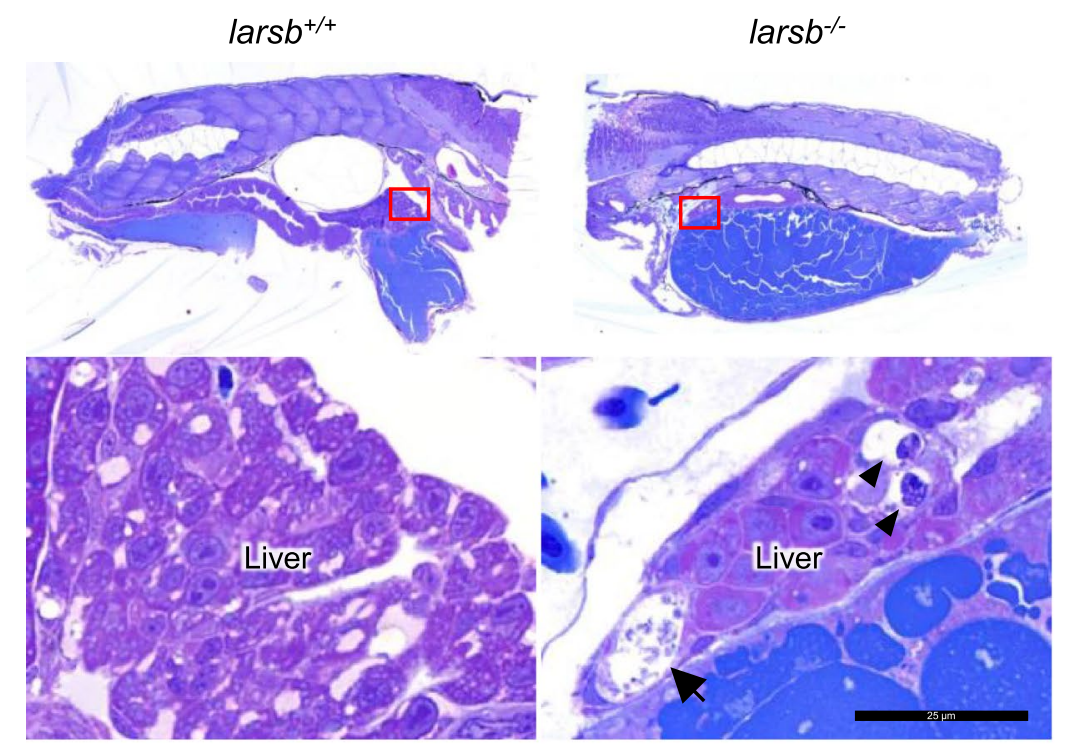

B

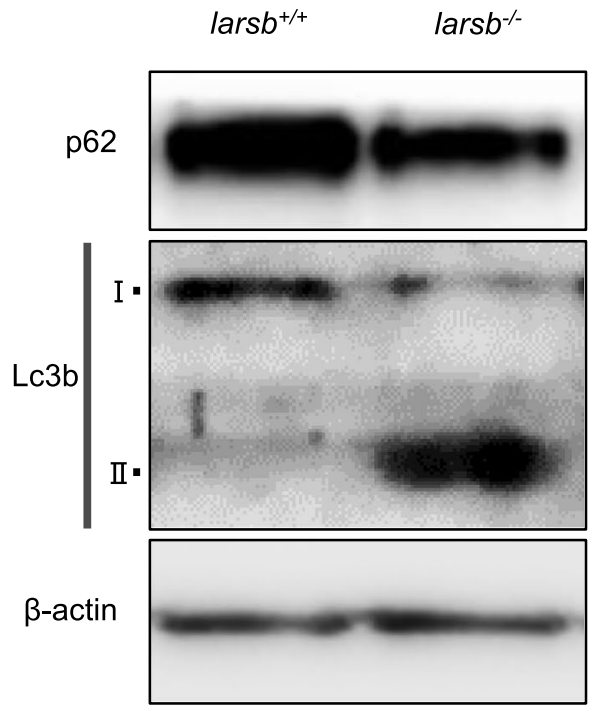

C

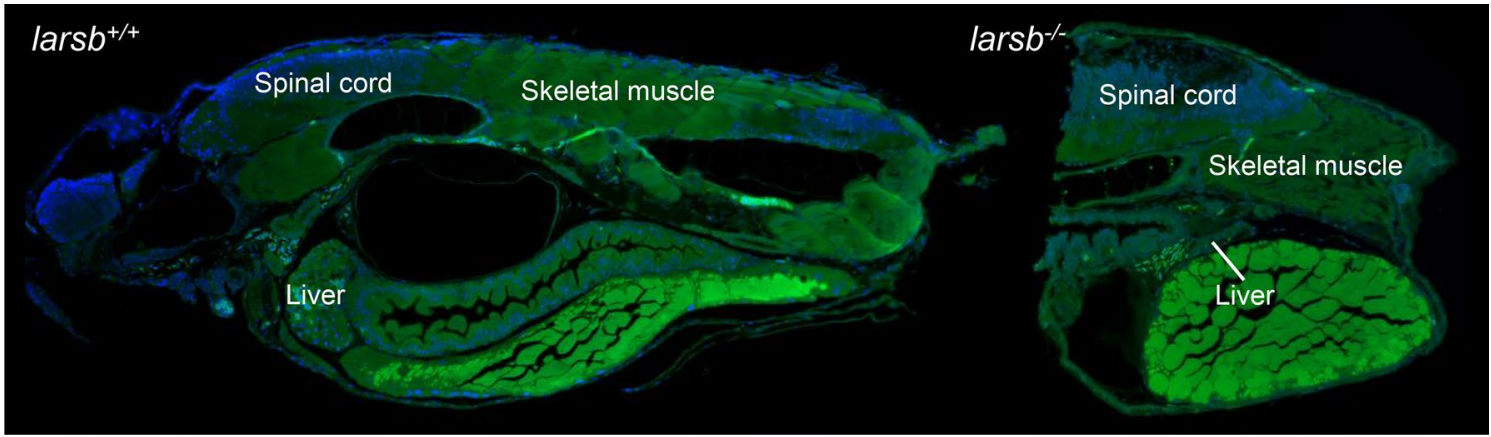

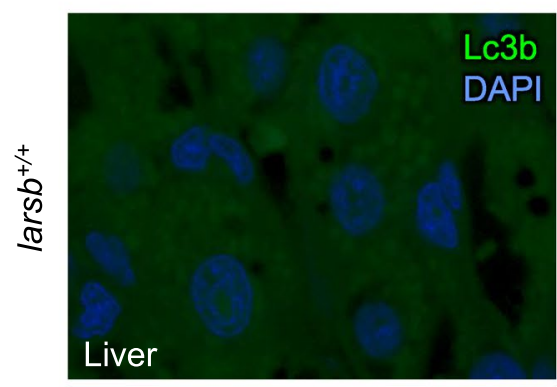

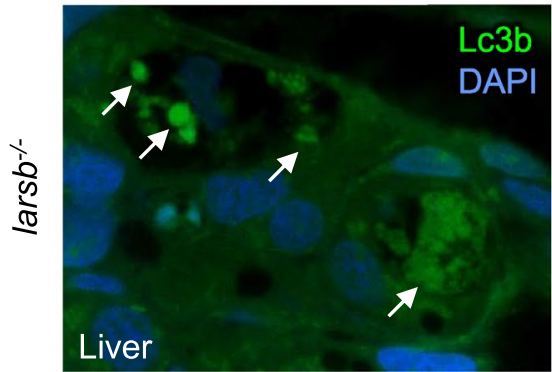

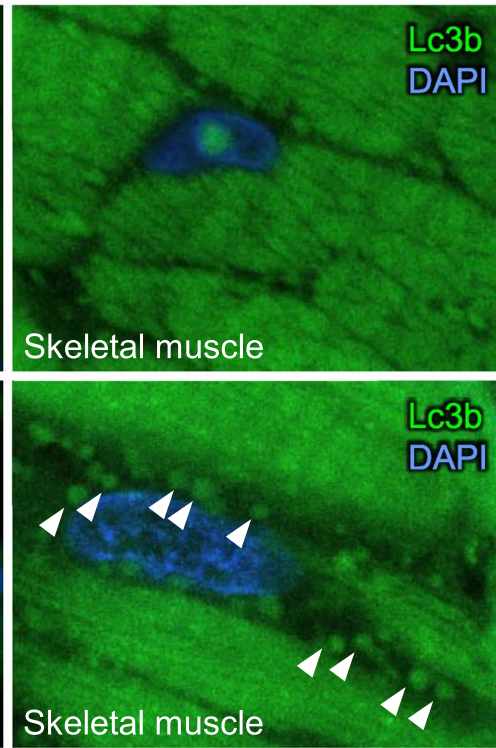

Lc3b DAPI
Spinal cord

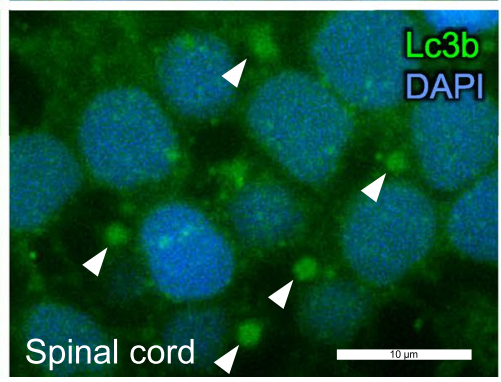


४Figure 3. Histopathology and fluorescent immunostaining of larsb-knockout larvae. (A) Lower magnification sagittal views (top row) and higher magnification views (bottom row) of lars $b^{+/+}$and $l a r s b^{-/-}$larvae. Huge vacuolations, which seemed to disappear in the cytoplasm, were seen in the livers of lars $b^{-/-}$larvae (black arrows), and some large vacuolations included a bare nucleus (black arrowheads). Scale bar: $25 \mu \mathrm{m}$. (B) Western blot analysis of p62 and Lc3b protein expression in $l a r s b^{+/+}$and lars $b^{-/-}$larvae. $\beta$-actin levels served as the loading control. (C) Lower magnification sagittal views of $l a r s b^{+/+}$and lars $b^{-/-}$larvae (top row). Fluorescent immunostaining against Lc3b (green) and DAPI (blue) of the livers, skeletal muscles, and spinal cords of larsb $b^{+/+}$ and $l a r s b^{-/-}$larvae. Livers of lars $b^{-/}$larvae had large vacuoles, including floating nuclei and various sized dots with Lc3b immunoreactivity (white arrows). Skeletal muscles and spinal cords of lars $b^{-/-}$larvae had many dots with Lc3b immunoreactivity (white arrowheads). Scale bar: $10 \mu \mathrm{m}$. Lars: leucyl-tRNA synthetase; Lc3b: microtubule-associated protein 1A/1B-light chain 3; DAPI: 4',6-diamidino-2-phenylindole.

ance with the ARRIVE guidelines. The study protocol was approved by the Institutional Review Board of Oita University (approval no. 180506).

Generation of the lars $^{-/-}$zebrafish line. A larsb $^{-/-}$zebrafish line was generated via CRISPR/ Cas9 gene editing ${ }^{18,19}$. The site of the lars $b$ sgRNA target was $5^{\prime}$-CAGTGTGCCGTCAGATGCACCGG-3', in the editing domain of the LARS protein. Cas 9 protein $(300 \mathrm{pg})$ and gRNA $(30 \mathrm{pg})$ were injected into one-cell-stage wildtype embryos. The mutation at the target site was verified via Sanger sequencing. The injected embryos were raised until adulthood and outcrossed with wild-type adults. DNA extracted from the F1 generation of whole larvae at $24 \mathrm{~h}$ post fertilization (hpf) was screened for indels by the heteroduplex mobility assay ${ }^{43,44}$ and Sanger sequencing. The F0 founder with germline transmission was selected to establish the knockout zebrafish line. F1 generations were raised to adulthood, had their fins clipped, and were sequenced. Fish carrying the same mutation (deletion of CACCG) were identified. All experiments were performed on embryos from the F2 or F3 progeny.

Generation of transgenic zebrafish. $\operatorname{Tg}[$ fabp10:mCherry] fish expressing mCherry exclusively in hepatocytes were generated using MultiSite Gateway ${ }^{\text {Tn }}$ kit (Thermo Fisher Scientific, Waltham, MA, USA) to produce vectors with Tol 2 transposon sites ${ }^{45}$. A 2.8 -kb promoter of the fabp 10 gene $^{23}$ was cloned into the p5E-mcs vector. Multisite Gateway cloning ${ }^{46}$ was performed with the destination vector pDestTol2pA2, the $5^{\prime}$ entry vector containing the fabp10 promoter, the middle entry vector containing pME-mCherry, and the $3^{\prime}$ entry vector containing p3E-polyA. DNA constructs ( $25 \mathrm{pg}$ ) and Tol2 mRNA (25 pg) were injected into wild-type zebrafish embryos at the one-cell stage.

Western blotting. Western blotting was performed with antibodies against Lars (\#13868; Cell Signaling Technology, Beverly, MA, USA), p62 (PM045; Medical \& Biological Laboratories, Nagoya, Japan), LC3B (PM036; Medical \& Biological Laboratories), ATG5 (NB110-53818; Novus Biologicals, Littleton, CO, USA), $\beta$-actin (A3854; Sigma-Aldrich, St. Louis, MO, USA), and glyceraldehyde 3-phosphate dehydrogenase (GAPDH) (G9295; Sigma-Aldrich). Samples for western blotting were lysed with lysis buffer $(0.5 \%$ NP-40, $10 \%$ glycerin, $50 \mathrm{mM}$ HEPES-KOH (pH 7.8), $150 \mathrm{mM} \mathrm{NaCl}$, and $1 \mathrm{mM}$ EDTA) with protease and phosphatase inhibitor cocktail (Thermo Fisher Scientific). Total proteins were separated by SDS-PAGE, transferred to Immobilon-P membranes (Millipore, Billerica, MA, USA), and probed with the above-mentioned antibodies. Densitometric analysis was performed using Fusion CAPT Advance software version 17.02 (Vilber Lourmat, Collegien, France; https://www.vilber.com/fusion-fx/).

Reverse-transcription quantitative polymerase chain reaction (RT-qPCR). The expression of lars-related protein genes was analysed using a reverse-transcription quantitative polymerase chain reaction (RT-qPCR). Total RNA was isolated from larvae at $6 \mathrm{dpf}$ using the RNAiso Plus reagent (Takara, Otsu, Japan), as per the manufacturer's protocol. First-strand cDNA was generated from $0.2 \mu \mathrm{g}$ RNA using the ReverTra Ace qPCR RT Master Mix with gDNA Remover (Toyobo, Osaka, Japan). After reverse transcription, RT-qPCR was performed using the FastStart Universal SYBR Green Master kit (Roche, Mannheim, Germany) on a LightCycler 96 (Roche), according to the manufacturer's protocol. The following primers were used for zebrafish RT-qPCR: lars $a$ and lars $b$ (forward), 5'-CAGACAGGAGAGGGAGTTGG-3'; larsb (reverse), 5'-GCAGGGCAT AAATGGTCTTG-3'; larsa (reverse), 5'-TGCAGCTGAAGCATTTAGGA-3'; lars2 (forward), 5'-CCCGTCACA CTGCCTAAAAT-3'; lars2 (reverse), 5'-GAACCAGCAGCTTCCTGAAC-3'; $\beta$-actin (forward), 5'-CGAGCT GTCTTCCCATCCA-3'; $\beta$-actin (reverse), 5'-TCACCAACGTAGCTGTCTTTCTG-3'.

O-dianisidine staining. The embryos at $72 \mathrm{hpf}$ were incubated in o-dianisidine staining buffer $(0.6 \mathrm{mg} /$ $\mathrm{mL}$ o-dianisidine, $10 \mathrm{mM}$ sodium acetate, $0.65 \%$ hydrogen peroxide, and $40 \%$ ethanol) for $15 \mathrm{~min}$ in the dark.

Morphological analyses. Zebrafish larvae were placed in 3\% methylcellulose, and images were acquired using a Leica M205 FA fluorescent stereo microscope. The liver size was measured manually using ImageJ software (1.52a) (Bethesda, MD, USA; https://imagej.nih.gov/ij/). For the microcephaly assay, the total body length and head diameter through the rear third of the eye lens ratio was measured with LAS X (Leica) and calculated as an index of microcephaly ${ }^{47,48}$. 

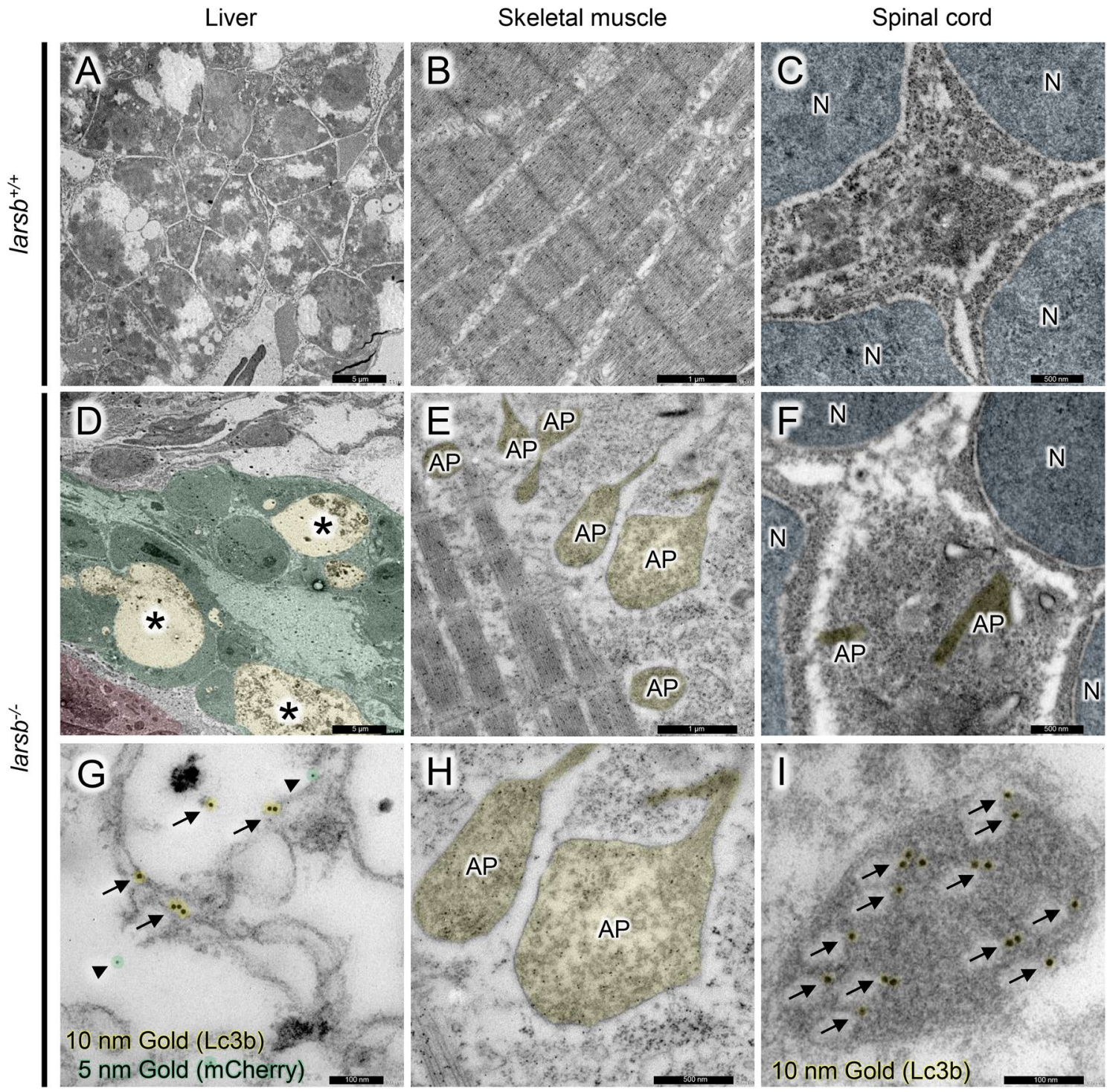

Figure 4. Immunoelectron microscopy of larsb-knockout larvae under $\operatorname{Tg}[$ fabp10:mcherry] background. (A-C) Immunoelectron microscopy of the liver, skeletal muscle, and spinal cord of larsb ${ }^{+/}$larva. (D-I) Immunoelectron microscopy of the liver, skeletal muscle, and spinal cord of lars $b^{-/-}$larva. The bottom row shows higher magnification images (G-I). Large vacuoles in the livers of lars $^{-/-}$larvae (asterisks) were composed of numerous irregular membranous structures, which showed immunoreactivity against both Lc3b (black arrows) and mCherry (black arrowheads) (D,G). Scale bar: $5.0 \mu \mathrm{m}$ for $(\mathbf{A}, \mathbf{D}) ; 1.0 \mu \mathrm{m}$ for B and E; $500 \mathrm{~nm}$ for (C,F,H); $100 \mathrm{~nm}$ for (G,I). AP: autophagosome, N: nucleus, Larsb: leucyl-tRNA synthetase, Lc3b: microtubule-associated protein 1A/1B-light chain 3.

Zebrafish survival analysis. Embryos were generated and housed at $28.5^{\circ} \mathrm{C}$. Larvae were transferred to rotifer feeding solution at $5 \mathrm{dpf}$, and the solution was replaced daily with additional rotifer feeding solution. The dishes were monitored twice a day until $12 \mathrm{dpf}$.

Zebrafish locomotion analysis. Locomotion was recorded and analysed as described in Yatsuka et al ${ }^{47}$. The trajectory plot data were recorded with a Visualix STD1 digital camera (Visualix, Kobe, Japan) attached to a Leica M80 microscope. The trajectory plot data were analysed using SMART video tracking software version 3.0.06 (PanLab, Harvard Apparatus, MA, USA; https://www.panlab.com/en/products/smart-video-track ing-software-panlab). Larvae at $6 \mathrm{dpf}$ were placed into 12 -well plates (one larva/well) in $1000 \mu \mathrm{l}$ embryo medium ( $0.03 \%$ saltwater). The plate was placed under the Leica M80 microscope and tracked as follows: 30 min adaptation and 10 min tracking. All data of the locomotion analysis were recorded and analysed with the Zantiks MWP (Zantiks, Cambridge, UK). Larvae at $6 \mathrm{dpf}$ were placed into 12-well plates (one larva/well) in $1000 \mu \mathrm{l}$ embryo medium. The plate was transferred to the Zantiks MWP and tracked as follows: 30 min adaptation and $10 \mathrm{~min}$ tracking. 
Histopathological staining and fluorescent immunostaining. Small larvae specimens were fixed with $0.1 \%$ glutaraldehyde in $4 \%$ paraformaldehyde for approximately $48 \mathrm{~h}$, and washed with phosphate-buffered saline. Then, the specimens were washed with gradually increasing concentrations of dimethylformamide and embedded in LR White resin (London Resin Company, Berkshire, UK). Histological examinations were performed using semi-thin sections $(1 \mu \mathrm{m}$ thick) and stained with toluidine blue dye. A double-labelling immunofluorescence analysis was performed on the semi-thin sections using the following primary antibody: rabbit polyclonal LC3B antibody (ab51520; Abcam, Cambridge, UK; 1:100). The secondary antibody used was Alexa Fluor 488 goat anti-rabbit IgG (A31627; Molecular Probes, Eugene, OR, USA; 1:500). Vectashield DAPI (H-1200-10; Vector Laboratories, Brussels, Belgium) was used as a nuclear marker. A laser scanning confocal microscope (BZ-X800, Keyence, Osaka, Japan) equipped with a $\times 100$ oil immersion objective was used to visualize immunoreactivity.

Immunoelectron microscopy. The ultrastructural localization of LC3B was examined using zebrafish larvae, employing the post-embedding method as described previously ${ }^{49,50}$. Small larvae specimens embedded in LR White Resin, prepared as semi-thin sections, were used. The RFP antibody was used for the detection of mCherry protein, because it reacts with RFP and other RFP variants, such as mCherry. Ultra-thin sections (70 $\mathrm{nm}$ thick) were cut, incubated with a rabbit polyclonal LC3B antibody (1:300) and a mouse monoclonal RFP antibody $(1: 100)$ for $2 \mathrm{~h}$ at $24{ }^{\circ} \mathrm{C}$, and reacted with 10-nm gold colloidal particle-conjugated anti-rabbit IgG (EMGFAR10; British BioCell International, Cardiff, UK; 1:30) and 5-nm gold colloidal particle-conjugated antimouse IgG (EMGMHL5; British BioCell International; 1:30). Finally, the sections were stained with lead citrate and examined using a JEM-1400 electron microscope at $80 \mathrm{kV}$ (JEOL, Tokyo, Japan).

Morpholino oligonucleotide injection. Morpholino oligonucleotide for atg5 (5'-CATCCTTGTCAT CTGCCATTATCAT-3') was obtained from Gene-Tools, LLC (Philomath, OR, USA). The atg5 morpholino oligo was used to inhibit $\operatorname{atg} 5$ translation by binding to atg 5 initiation sites ${ }^{29}$. Atg 5 morpholino oligo or control morpholino oligo $(0.02 \mathrm{pmol})$ was injected into the zebrafish eggs at the one-cell stage.

Bafilomycin A1, chloroquine, 3-methyladenine, and rapamycin treatments. Embryos were treated with bafilomycin A1 (2.5 nM; EMD Millipore, Darmstadt, Germany), chloroquine (10 nM; SigmaAldrich), 3-methyladenine (5 mM; Sigma-Aldrich), rapamycin (5 $\mu \mathrm{M}$; LC Laboratories, Woburn, MA, USA), or dimethyl sulfoxide (DMSO) as the control, in embryo medium from 48 to $72 \mathrm{hpf}$ for morphological experiments and in the larval stage from 4 to $13 \mathrm{dpf}$ for survival experiments. Embryos were treated with rapamycin $(5 \mu \mathrm{M}$; LC Laboratories, Woburn, MA, USA) or DMSO in embryo medium from 60 to $96 \mathrm{hpf}$ for the morphological experiments ${ }^{17}$ and in the larval stage from 4 to $13 \mathrm{dpf}$ for the survival experiments. The water containing the drug was replaced daily.

Statistics. Statistical analyses were performed using GraphPad Prism software version 8 (GraphPad Software, Inc., San Diego, CA, USA; https://www.graphpad.com/scientific-software/prism/). All values are expressed as mean \pm SEM. Comparisons between groups were made by Student's $t$-test. Statistical difference for survival curves were analysed using a Log-rank (Mantel-Cox) test. $\mathrm{P}<0.05$ was considered statistically significant. 
A

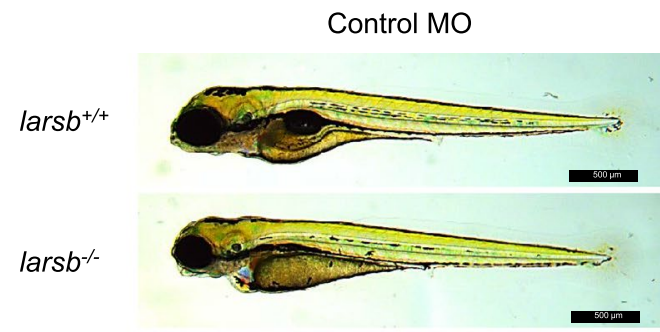

B

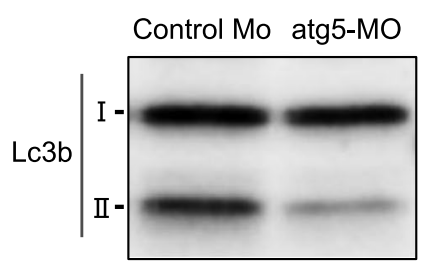

$\beta$-actin

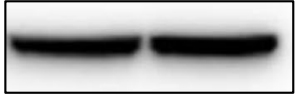

E

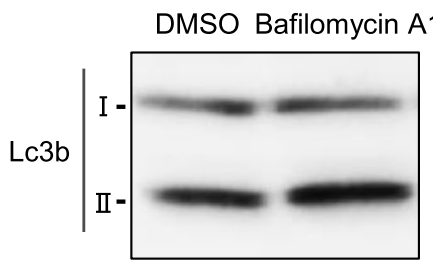

$\beta$-actin

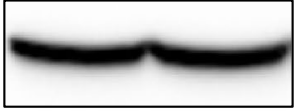

C

Control $\mathrm{MO}$

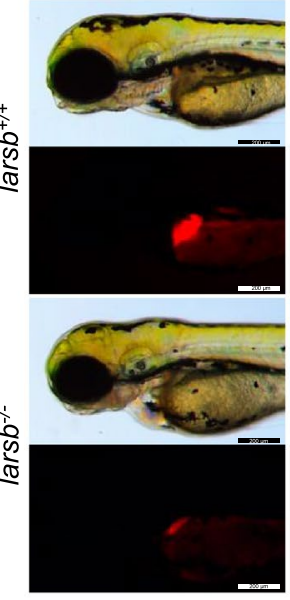

F

DMSO

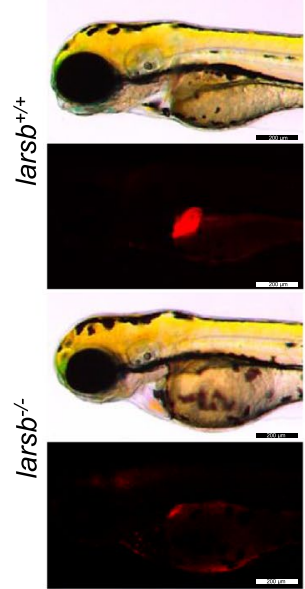

$\operatorname{atg} 5-\mathrm{MO}$

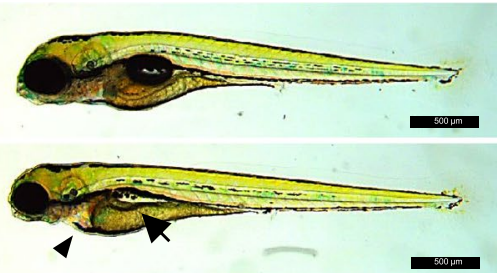

$\operatorname{atg} 5-\mathrm{MO}$

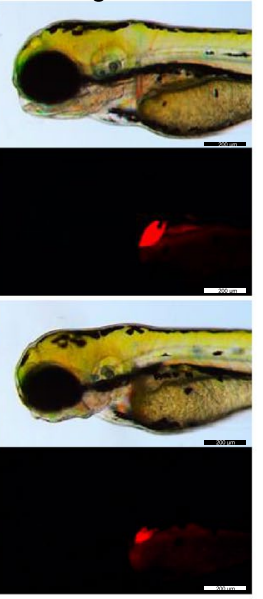

Bafilomycin A1
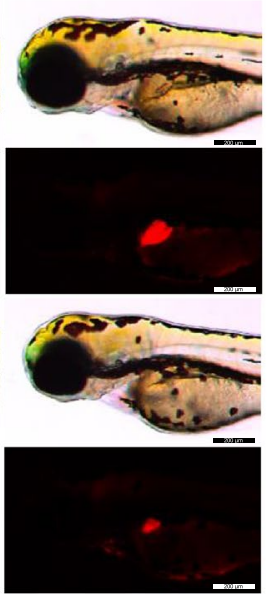

D

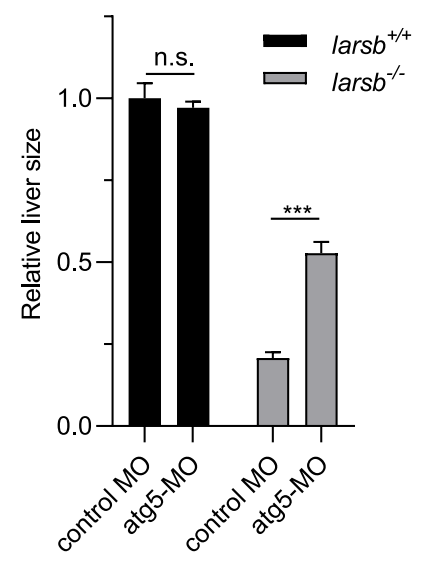

G

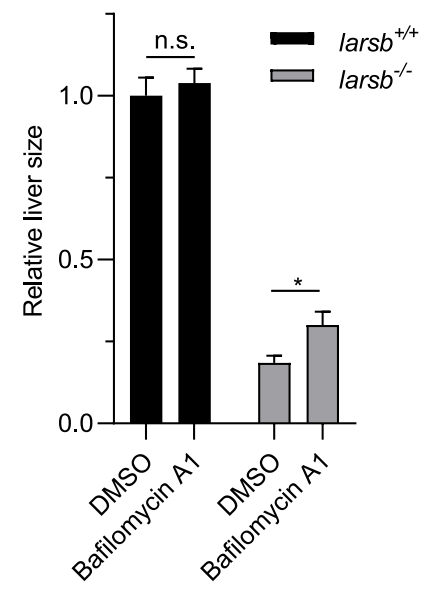

$\mathrm{H}$

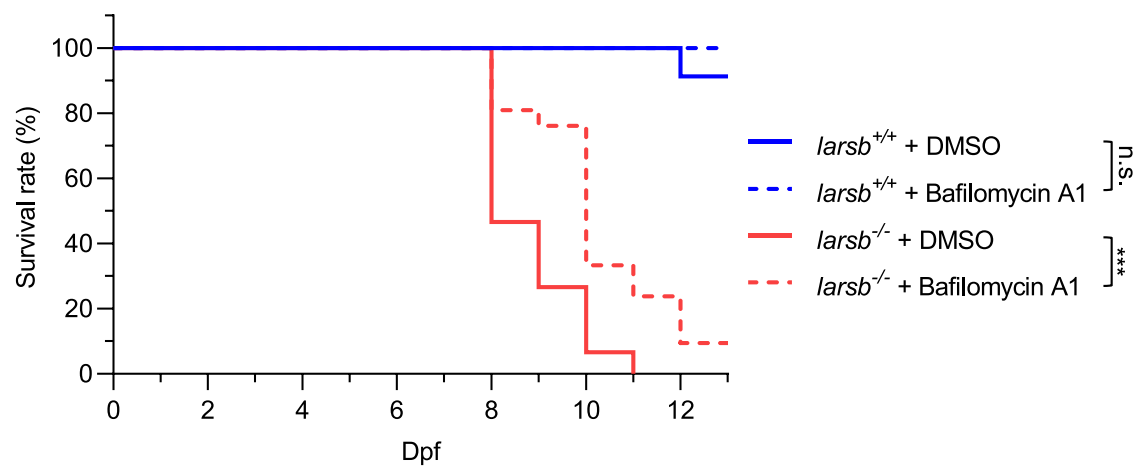


4Figure 5. Inhibition of autophagy prevents abnormal development and improves survival in lars $b$-knockout larvae. (A) Morphology of lars $b^{+/+}$and larsb $^{-/-}$embryos injected with either control MO or atg5-MO (72 h post fertilization (hpf)). Scale bars: $500 \mu \mathrm{m}$. (B) Western blot analysis of Lc3b protein expression at $72 \mathrm{hpf}$ for wild-type embryos injected with either control MO or atg5-MO. $\beta$-actin levels served as the loading control. (C) Morphological abnormality at $72 \mathrm{hpf}$ in the livers of lars ${ }^{-/}$larvae under $\operatorname{Tg}[$ fabp10:mcherry] background injected with either control MO or atg5-MO. Scale bars: $200 \mu \mathrm{m}$. (D) Quantification of liver size in lars $b^{-/-}$ larvae under $\operatorname{Tg}[$ fabp10:mcherry] background (72 hpf). Liver sizes were evaluated using ImageJ software version 1.52a (https://imagej.nih.gov/ij/). $\mathrm{n}=4$ fish/group. Error bars indicate SEM. Student's t-test; ${ }^{\star * \star} \mathrm{P}<0.001$. (E) Western blot analysis of Lc3b protein expression at $72 \mathrm{hpf}$ for wild-type embryos treated with DMSO or bafilomycin A1. $\beta$-actin levels served as the loading control. (F) Morphological abnormality at 72 hpf in the livers of $l a r s b^{-/-}$larvae under Tg[fabp10:mcherry] background treated with DMSO or bafilomycin A1. Scale

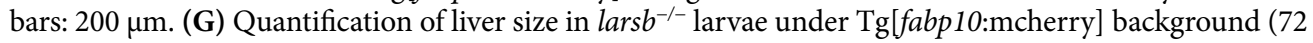
hpf). Liver sizes were evaluated using ImageJ software version 1.52a (https://imagej.nih.gov/ij/). $\mathrm{n}=10 \mathrm{fish} /$ group. Error bars indicate SEM. Student's t-test; ${ }^{\star} \mathrm{P}<0.05$. (H) Kaplan-Meier survival curve of lars $b^{+/+}(\mathrm{n}=23)$ and $\operatorname{lars}^{-/-}(\mathrm{n}=15)$ larvae treated with DMSO and $\operatorname{lars}^{+/+}(\mathrm{n}=11)$ and larsb $^{-/-}$larvae $(\mathrm{n}=21)$ treated with bafilomycin A1. Statistics were calculated and the figure was produced in GraphPad software version 8 (https:// www.graphpad.com/scientific-software/prism/). Larsb: leucyl-tRNA synthetase b, MO: morpholino, n.s.: nonsignificant, DMSO: dimethyl sulfoxide, Dpf: days post fertilization.

Received: 21 December 2020; Accepted: 5 April 2021

Published online: 16 April 2021

\section{References}

1. Schimmel, P. Aminoacyl tRNA synthetases: General scheme of structure-function relationships in the polypeptides and recognition of transfer RNAs. Annu. Rev. Biochem. 56, 125-158. https://doi.org/10.1146/annurev.bi.56.070187.001013 (1987).

2. Antonellis, A. \& Green, E. D. The role of aminoacyl-tRNA synthetases in genetic diseases. Annu. Rev. Genomics Hum. Genet. 9, 87-107. https://doi.org/10.1146/annurev.genom.9.081307.164204 (2008).

3. Yao, P. \& Fox, P. L. Aminoacyl-tRNA synthetases in medicine and disease. EMBO Mol. Med. 5, 332-343. https://doi.org/10.1002/ emmm.201100626 (2013).

4. Meyer-Schuman, R. \& Antonellis, A. Emerging mechanisms of aminoacyl-tRNA synthetase mutations in recessive and dominant human disease. Hum. Mol. Genet. 26, R114-R127. https://doi.org/10.1093/hmg/ddx231 (2017).

5. Han, J. M. et al. Leucyl-tRNA synthetase is an intracellular leucine sensor for the mTORC1-signaling pathway. Cell 149, 410-424. https://doi.org/10.1016/j.cell.2012.02.044 (2012).

6. Bonfils, G. et al. Leucyl-tRNA synthetase controls TORC1 via the EGO complex. Mol. Cell 46, 105-110. https://doi.org/10.1016/j. molcel.2012.02.009 (2012).

7. He, C. \& Klionsky, D. J. Regulation mechanisms and signaling pathways of autophagy. Annu. Rev. Genet. 43, 67-93. https://doi. org/10.1146/annurev-genet-102808-114910 (2009).

8. Kim, J. H. et al. Control of leucine-dependent mTORC1 pathway through chemical intervention of leucyl-tRNA synthetase and RagD interaction. Nat. Commun. 8, 732. https://doi.org/10.1038/s41467-017-00785-0 (2017).

9. Park, S. G., Ewalt, K. L. \& Kim, S. Functional expansion of aminoacyl-tRNA synthetases and their interacting factors: New perspectives on housekeepers. Trends Biochem. Sci. 30, 569-574. https://doi.org/10.1016/j.tibs.2005.08.004 (2005).

10. Guo, M. \& Schimmel, P. Essential nontranslational functions of tRNA synthetases. Nat Chem Biol 9, 145-153. https://doi.org/10. 1038/nchembio.1158 (2013).

11. van Meel, E. et al. Rare recessive loss-of-function methionyl-tRNA synthetase mutations presenting as a multi-organ phenotype. BMC Med. Genet. 14, 106. https://doi.org/10.1186/1471-2350-14-106 (2013).

12. Kopajtich, R. et al. Biallelic IARS mutations cause growth retardation with prenatal onset, intellectual disability, muscular hypotonia, and infantile hepatopathy. Am. J. Hum. Genet. 99, 414-422. https://doi.org/10.1016/j.ajhg.2016.05.027 (2016).

13. Nowaczyk, M. J. et al. A novel multisystem disease associated with recessive mutations in the tyrosyl-tRNA synthetase (YARS) gene. Am. J. Med. Genet. A 173, 126-134. https://doi.org/10.1002/ajmg.a.37973 (2017).

14. Fuchs, S. A. et al. Aminoacyl-tRNA synthetase deficiencies in search of common themes. Genet. Med. 21, 319-330. https://doi. org/10.1038/s41436-018-0048-y (2019).

15. Casey, J. P. et al. Clinical and genetic characterisation of infantile liver failure syndrome type 1, due to recessive mutations in LARS. J. Inherit. Metab. Dis. 38, 1085-1092. https://doi.org/10.1007/s10545-015-9849-1 (2015).

16. Lenz, D. et al. Genotypic diversity and phenotypic spectrum of infantile liver failure syndrome type 1 due to variants in LARS1. Genet. Med. https://doi.org/10.1038/s41436-020-0904-4 (2020).

17. Wang, Z., Song, J., Luo, L. \& Ma, J. Loss of Leucyl-tRNA synthetase b leads to ILFS1-like symptoms in zebrafish. Biochem. Biophys. Res. Commun. 505, 378-384. https://doi.org/10.1016/j.bbrc.2018.09.133 (2018).

18. Hruscha, A. et al. Efficient CRISPR/Cas9 genome editing with low off-target effects in zebrafish. Development 140, $4982-4987$. https://doi.org/10.1242/dev.099085 (2013).

19. Hwang, W. Y. et al. Efficient genome editing in zebrafish using a CRISPR-Cas system. Nat. Biotechnol. 31, 227-229. https://doi. org/10.1038/nbt.2501 (2013).

20. Peroutka, C. et al. Severe neonatal manifestations of infantile liver failure syndrome type 1 caused by cytosolic leucine-tRNA synthetase deficiency. JIMD Rep. 45, 71-76. https://doi.org/10.1007/8904_2018_143 (2019).

21. El-Brolosy, M. A. et al. Genetic compensation triggered by mutant mRNA degradation. Nature 568, 193-197. https://doi.org/10. 1038/s41586-019-1064-z (2019).

22. Ma, Z. et al. PTC-bearing mRNA elicits a genetic compensation response via Upf3a and COMPASS components. Nature 568, 259-263. https://doi.org/10.1038/s41586-019-1057-y (2019).

23. Her, G. M., Chiang, C. C., Chen, W. Y. \& Wu, J. L. In vivo studies of liver-type fatty acid binding protein (L-FABP) gene expression in liver of transgenic zebrafish (Danio rerio). FEBS Lett. 538, 125-133. https://doi.org/10.1016/s0014-5793(03)00157-1 (2003).

24. Her, G. M., Yeh, Y. H. \& Wu, J. L. 435-bp liver regulatory sequence in the liver fatty acid binding protein (L-FABP) gene is sufficient to modulate liver regional expression in transgenic zebrafish. Dev. Dyn. 227, 347-356. https://doi.org/10.1002/dvdy.10324 (2003).

25. Ognjenovic, J. \& Simonovic, M. Human aminoacyl-tRNA synthetases in diseases of the nervous system. RNA Biol. 15, 623-634. https://doi.org/10.1080/15476286.2017.1330245 (2018).

26. Liu, Y. \& Levine, B. Autosis and autophagic cell death: the dark side of autophagy. Cell Death Differ. 22, 367-376. https://doi.org/ $10.1038 /$ cdd.2014.143 (2015). 
27. Bai, H., Inoue, J., Kawano, T. \& Inazawa, J. A transcriptional variant of the LC3A gene is involved in autophagy and frequently inactivated in human cancers. Oncogene 31, 4397-4408. https://doi.org/10.1038/onc.2011.613 (2012).

28. Matsushita, M. et al. Structure of Atg5.Atg16, a complex essential for autophagy. J. Biol. Chem. 282, 6763-6772, https://doi.org/ 10.1074/jbc.M609876200 (2007).

29. Hu, Z., Zhang, J. \& Zhang, Q. Expression pattern and functions of autophagy-related gene atg5 in zebrafish organogenesis. Autophagy 7, 1514-1527. https://doi.org/10.4161/auto.7.12.18040 (2011).

30. Mizushima, N. \& Yoshimori, T. How to interpret LC3 immunoblotting. Autophagy 3, 542-545. https://doi.org/10.4161/auto.4600 (2007).

31. Chicote, J., Yuste, V. J., Boix, J. \& Ribas, J. Cell death triggered by the autophagy inhibitory drug 3-methyladenine in growing conditions proceeds with DNA damage. Front. Pharmacol. 11, 580343. https://doi.org/10.3389/fphar.2020.580343 (2020).

32. Sucularli, C. et al. Functionally conserved effects of rapamycin exposure on zebrafish. Mol Med Rep 13, 4421-4430. https://doi. org/10.3892/mmr.2016.5059 (2016).

33. Naito, T., Kuma, A. \& Mizushima, N. Differential contribution of insulin and amino acids to the mTORC1-autophagy pathway in the liver and muscle. J. Biol. Chem. 288, 21074-21081. https://doi.org/10.1074/jbc.M113.456228 (2013).

34. Matsushima, Y. \& Kaguni, L. S. Differential phenotypes of active site and human autosomal dominant progressive external ophthalmoplegia mutations in Drosophila mitochondrial DNA helicase expressed in Schneider cells. J. Biol. Chem. 282, 9436-9444. https://doi.org/10.1074/jbc.M610550200 (2007).

35. Fujioka, Y., Noda, N. N., Nakatogawa, H., Ohsumi, Y. \& Inagaki, F. Dimeric coiled-coil structure of Saccharomyces cerevisiae Atg16 and its functional significance in autophagy. J. Biol. Chem. 285, 1508-1515. https://doi.org/10.1074/jbc.M109.053520 (2010).

36. Mizushima, N., Yoshimori, T. \& Levine, B. Methods in mammalian autophagy research. Cell 140, 313-326. https://doi.org/10. 1016/j.cell.2010.01.028 (2010).

37. Lee, E. et al. Autophagy is essential for cardiac morphogenesis during vertebrate development. Autophagy 10, 572-587. https:// doi.org/10.4161/auto.27649 (2014).

38. Yuan, N. et al. Bafilomycin A1 targets both autophagy and apoptosis pathways in pediatric B-cell acute lymphoblastic leukemia. Haematologica 100, 345-356. https://doi.org/10.3324/haematol.2014.113324 (2015).

39. Yan, Y. et al. Bafilomycin A1 induces caspase-independent cell death in hepatocellular carcinoma cells via targeting of autophagy and MAPK pathways. Sci. Rep. 6, 37052. https://doi.org/10.1038/srep37052 (2016).

40. Sang, Z., Zhang, P., Wei, Y. \& Dong, S. miR-214-3p attenuates sepsis-induced myocardial dysfunction in mice by inhibiting autophagy through PTEN/AKT/mTOR pathway. Biomed. Res. Int. 2020, 1409038. https://doi.org/10.1155/2020/1409038 (2020).

41. Pickrell, A. M. \& Youle, R. J. The roles of PINK1, parkin, and mitochondrial fidelity in Parkinson's disease. Neuron 85, 257-273. https://doi.org/10.1016/j.neuron.2014.12.007 (2015).

42. Long, Z. et al. Dynamic changes of autophagic flux induced by Abeta in the brain of postmortem Alzheimer's disease patients, animal models and cell models. Aging (Albany NY) 12, 10912-10930. https://doi.org/10.18632/aging.103305 (2020).

43. Kostrikis, L. G. et al. Genetic analysis of human immunodeficiency virus type 1 strains from patients in Cyprus: Identification of a new subtype designated subtype I. J. Virol. 69, 6122-6130 (1995).

44. Ota, S. et al. Efficient identification of TALEN-mediated genome modifications using heteroduplex mobility assays. Genes Cells 18, 450-458. https://doi.org/10.1111/gtc.12050 (2013).

45. Kwan, K. M. et al. The Tol2kit: a multisite gateway-based construction kit for Tol2 transposon transgenesis constructs. Dev. Dyn. 236, 3088-3099. https://doi.org/10.1002/dvdy.21343 (2007).

46. Hartley, J. L., Temple, G. F. \& Brasch, M. A. DNA cloning using in vitro site-specific recombination. Genome Res. 10, $1788-1795$. https://doi.org/10.1101/gr.143000 (2000).

47. Yatsuka, H. et al. Exosc2 deficiency leads to developmental disorders by causing a nucleotide pool imbalance in zebrafish. Biochem. Biophys. Res. Commun. 533, 1470-1476. https://doi.org/10.1016/j.bbrc.2020.10.044 (2020).

48. Braun, D. A. et al. Mutations in KEOPS-complex genes cause nephrotic syndrome with primary microcephaly. Nat. Genet. 49, 1529-1538. https://doi.org/10.1038/ng.3933 (2017).

49. Miyahara, H. et al. Suppressed expression of autophagosomal protein LC3 in cortical tubers of tuberous sclerosis complex. Brain Pathol. 23, 254-262. https://doi.org/10.1111/j.1750-3639.2012.00634.x (2013).

50. Miyahara, H. et al. Neuronal differentiation associated with Gli3 expression predicts favorable outcome for patients with medulloblastoma. Neuropathology 34, 1-10. https://doi.org/10.1111/neup.12052 (2014).

\section{Acknowledgements}

We thank M. Nakamura-Ota, K. Shimizu, and M. Iwao for their excellent technical assistance. TH was supported by the Japan Society for the Promotion of Science (20H03644), the Takeda Science Foundation, the Kamizono Kids Clinic, and the Mizoguchi Urology Clinic. MI was supported by the Japan Society for the Promotion of Science (19K17366).

\section{Author contributions}

M.I. generated mutant zebrafish and performed zebrafish phenotyping with the assistance of M.T., K.K., and M.M. H.M. performed the histological analysis. H.S. and N.S. performed the biochemical assays. T.I. and R.H. provided key reagents and technical assistance for the generation of mutant zebrafish. R.U. performed the locomotor activity analysis. K.I. and T.H. coordinated the project and wrote the manuscript. All authors reviewed the manuscript.

\section{Competing interests}

The authors declare no competing interests.

\section{Additional information}

Supplementary Information The online version contains supplementary material available at https://doi.org/ 10.1038/s41598-021-87879-4.

Correspondence and requests for materials should be addressed to K.I. or T.H.

Reprints and permissions information is available at www.nature.com/reprints.

Publisher's note Springer Nature remains neutral with regard to jurisdictional claims in published maps and institutional affiliations. 
(c) (i) Open Access This article is licensed under a Creative Commons Attribution 4.0 International cc) License, which permits use, sharing, adaptation, distribution and reproduction in any medium or format, as long as you give appropriate credit to the original author(s) and the source, provide a link to the Creative Commons licence, and indicate if changes were made. The images or other third party material in this article are included in the article's Creative Commons licence, unless indicated otherwise in a credit line to the material. If material is not included in the article's Creative Commons licence and your intended use is not permitted by statutory regulation or exceeds the permitted use, you will need to obtain permission directly from the copyright holder. To view a copy of this licence, visit http://creativecommons.org/licenses/by/4.0/.

(C) The Author(s) 2021 